\title{
Atrial Fibrillation and Coronary Artery Disease: Deciding on The Best Antithrombotic Regimen
}

Jason G. Andrade MD, Marc W. Deyell MD MSc, Laurent Macle MD

DOI: 10.22374/cjgim.v13iSP1.309

\section{About the Authors}

Jason Andrade and Laurent Macle are with the Electrophysiology Service at the Montreal Heart Institute and the Department of Medicine, Université de Montréal, Montreal, Canada. Jason Andrade and Marc Deyell are is with the Heart Rhythm Services, Department of Medicine, The University of British Columbia, British Columbia, Canada.

Correspondence may be directed to: Jason.andrade@vch.ca

\begin{abstract}
Atrial fibrillation (AF) is a chronic progressive disease characterized by exacerbations and remissions. Up to $20-30 \%$ of patients with AF also have coronary artery disease (CAD). In patients with concomitant $\mathrm{AF}$ and $\mathrm{CAD}$, the management of antithrombotic therapy is challenging. Oral anticoagulation (OAC) is indicated for the prevention of AF-related stroke and systemic embolism, whereas antiplatelet therapy is indicated for the prevention of coronary events. Each of these therapeutic avenues offers a relative efficacy benefit (e.g., dual antiplatelet therapy [DAPT] is more effective than OAC alone in reducing cardiovascular death, myocardial infarction, stent thrombosis, and ischemic coronary events in an ACS population), but with a relative compromise (e.g., DAPT is significantly inferior to OAC for the prevention of stroke/ systemic embolism in an AF population at increased risk of AF-related stroke). The purpose of this review is to explore the current evidence and rationale for antithrombotic treatment strategies in patients with both AF and CAD.
\end{abstract}

Atrial fibrillation (AF) is the most common sustained arrhythmia, and represents a major burden to our healthcare system. Current evidence indicates that the prevalence of $\mathrm{AF}$ is in the range of $2 \%$ of the general population. ${ }^{1,2}$ Of those with AF, up to $20-30 \%$ have concomitant coronary artery disease (CAD), and 5-15\% will require percutaneous coronary intervention (PCI). ${ }^{3,4}$

In patients with both $\mathrm{AF}$ and $\mathrm{CAD}$ the management of antithrombotic therapy can be challenging. Oral anticoagulation $(\mathrm{OAC})$ is indicated for the prevention of AF-related stroke and systemic embolism, whereas antiplatelet therapy is indicated for the prevention of coronary events. Each offers a relative efficacy benefit with either a relative efficacy compromise or a relative safety compromise (e.g., dual antiplatelet therapy [DAPT] is more effective than $\mathrm{OAC}$ alone in reducing ischemic coronary events in an ACS population but is significantly inferior to OAC alone for the prevention of stroke/systemic embolism in an $\mathrm{AF}$ population)..$^{5}$ As such clinicians have perceived an obligation to treat patients with concomitant AF and CAD using both OAC with and antiplatelet therapy in spite of the potential for increased risk of fatal and nonfatal bleeding events, including intracranial hemorrhage. ${ }^{6-8}$

The purpose of this review is to explore the current evidence and rationale for antithrombotic treatment strategies in $\mathrm{AF}$ patients at risk for AF-associated stroke (i.e., those aged $\geq 65$ years or CHADS2 score $\geq 1$ ) with concomitant CAD. Specifically, with a focus on the key clinical questions of: (1) What is the 
preferred antithrombotic therapeutic regimen for $\mathrm{AF}$ patients with ACS or undergoing PCI? (2) Should we use NOACs as part of the dual or triple therapy regimen? (3) What is the preferred P2Y12 inhibitor as part of the combination therapy regimen? (4) What is the optimal duration of triple antithrombotic therapy? (5) What is the optimal antithrombotic therapy for AF patients with stable CAD?

\section{Question 1 - What Is the Preferred Antithrombotic Therapeutic Regimen for af Patients With Acs or Undergoing Pci?}

The most complicated scenario for management of AF and CAD is the patient with a strong indication for OAC therapy for stroke prevention (aged $\geq 65$ years or CHADS2 score $\geq 1$ ), in whom an acute coronary event has occurred. In this circumstance the patient is at risk for both AF-related stroke/systemic embolism as well as adverse ischemic coronary events (e.g., stent thrombosis and recurrent myocardial infarction). There are three key clinical trials that have been performed in an effort to address the therapeutic dilemma associated with patients requiring oral anticoagulation and dual antiplatelet therapy (Table 1).

The "What Is the Optimal Antiplatelet and Anticoagulation Therapy in Patients With Oral Anticoagulation and Coronary Stenting" (WOEST) study randomized 573 patients with a need for anticoagulation undergoing PCI to dual pathway therapy (OAC and clopidogrel $75 \mathrm{mg} /$ day) or to triple antithrombotic therapy (OAC, clopidogrel, and aspirin $80 \mathrm{mg} /$ day). ${ }^{19,20}$ Treatment was continued for 1 month after BMS placement (35\% of patients) and for 1 year after DES placement (65\% of patients). The primary outcome of this open-label trial was any

Table 1. Relative Comparison of the WOEST, PIONEER AF-PCI, RE-DUAL Trials

\begin{tabular}{|c|c|c|c|}
\hline & Woest & Pioneer AF-PCI & Re-Dual PCI \\
\hline Design & Multicenter RCT & Multicenter RCT & Multicenter RCT \\
\hline Population & 573 & 2124 & 2725 \\
\hline Groups & $\begin{array}{c}W+A+C(284) \\
W+C(279)\end{array}$ & $\begin{array}{c}W+A+C(697) \\
R 15+C(696) \\
R 2.5+A+C(706)\end{array}$ & $\begin{array}{l}W+A+C(981) \\
D 110+C(981) \\
D 150+C(763)\end{array}$ \\
\hline Follow-up & 12 months & 12 months & 14 months \\
\hline Age in years & 68.7 & 70.1 & 70.8 \\
\hline Male & $74.4 \%$ & $74.4 \%$ & $80.0 \%$ \\
\hline$\% \mathrm{AF}$ & $69 \%$ & $100 \%$ & $100 \%$ \\
\hline \multicolumn{4}{|l|}{ Outcomes } \\
\hline - Any Bleeding & $\begin{array}{c}\text { HR } 0.36 \\
95 \% \mathrm{Cl} 0.26-0.50\end{array}$ & $\begin{array}{c}\mathrm{R} 15+\mathrm{C}-\mathrm{HR} 0.59 \\
95 \% \mathrm{Cl} 0.47-0.76 \\
\mathrm{R} 2.5+\mathrm{A}+\mathrm{C}-\mathrm{HR} 0.63 \\
95 \% \mathrm{Cl} 0.50-0.80\end{array}$ & $\begin{array}{l}\text { D110 HR 0.52** } \\
95 \% \mathrm{Cl} 0.42-0.63 \\
\text { D150 HR 0.72** } \\
95 \% \mathrm{Cl} 0.58-0.88\end{array}$ \\
\hline - Major TMI bleeding & $\begin{array}{c}\text { HR } 0.56 \\
95 \% \mathrm{Cl} 0.25-1.27\end{array}$ & $\begin{array}{c}\mathrm{R} 15+\mathrm{C}-\mathrm{HR} 0.66 \\
95 \% \mathrm{Cl} 0.33-1.31 \\
\mathrm{R} 2.5+\mathrm{A}+\mathrm{C}-\mathrm{HR} 0.57 \\
95 \% \mathrm{Cl} 0.28-1.16\end{array}$ & $\begin{array}{c}\text { D110 HR 0.37* } \\
95 \% \mathrm{Cl} 0.20-0.68 \\
\text { D150 HR } 0.51 \\
95 \% \mathrm{Cl} 0.28-0.93\end{array}$ \\
\hline - MACE & $\begin{array}{c}\text { HR } 0.6^{1} \\
95 \% \mathrm{Cl} 0.38-0.94\end{array}$ & $\begin{array}{c}\mathrm{R} 15+\mathrm{C}-\mathrm{HR} 1.08^{2} \\
95 \% \mathrm{Cl} 0.69-1.68 \\
\mathrm{R} 2.5+\mathrm{A}+\mathrm{C}-\mathrm{HR} 0.93^{2} \\
95 \% \mathrm{Cl} 0.59-1.48\end{array}$ & $\begin{array}{c}\text { D110 HR 1.13 } \\
95 \% \mathrm{Cl} 0.90-1.43 \\
\text { D150 HR 0.893 } \\
95 \% \mathrm{Cl} 0.67-1.19\end{array}$ \\
\hline
\end{tabular}

$\mathrm{W}=$ warfarin; $\mathrm{A}=\mathrm{ASA} 81 \mathrm{mg}$ daily; $\mathrm{C}=$ clopidogrel $75 \mathrm{mg}$ daily; $\mathrm{R} 15=$ rivaroxaban $15 \mathrm{mg}$ daily; $\mathrm{R} 2.5$ = rivaroxaban $2.5 \mathrm{mg}$ bid; $\mathrm{D} 110=$ dabigatran $110 \mathrm{mg}$ bid; $\mathrm{D} 150=$ dabigatran $150 \mathrm{mg}$ bid.

**ISTH and clinically relevant non-major bleeding.

${ }^{1}$ WOEST = death, myocardial infarction, stroke, target vessel revascularization, stent thrombosis.

${ }^{2}$ PIONEER AF-PCI = cardiovascular death, myocardial infarction, stroke.

${ }^{3} \mathrm{RE}-\mathrm{DUAL} \mathrm{PCl}=$ Death, revascularization, myocardial infarction, stroke, systemic thromboembolism. 
bleeding event within 1 year of follow-up (e.g., TIMI major plus minor). Dual pathway therapy was associated with a significant reduction in overall bleeding complications (19.4\% with DT vs. $44.4 \%$ with TT), without significant differences in major bleeds (3.2\% vs. $5.6 \%)$. Though not powered to detect differences in major thrombotic outcomes, the combined endpoint of major adverse cardiac and cerebrovascular events (MACCE - death, myocardial infarction, stroke, target vessel revascularization, stent thrombosis) was significantly reduced with dual pathway therapy ( $11.1 \%$ vs. 17.6$)$ as was all-cause mortality $(2.5 \%$ vs. $6.4 \% ; \mathrm{P}=0.027)$.

The "Prevention of bleeding in patients with AF undergoing PCI” (PIONEER AF-PCI) study randomized 2,124 patients with non-valvular AF undergoing PCI for ACS (51\%) or for stable CAD to receive, in a 1:1:1 ratio: (1) P2Y12 inhibitor (94\% clopidogrel) plus rivaroxaban $15 \mathrm{mg}$ daily (dual pathway) for 12 months (709 patients), (2) DAPT plus rivaroxaban $2.5 \mathrm{mg}$ twice daily (reduced dose triple antithrombotic therapy) for 1,6 or 12 months (709 patients), or (3) traditional triple antithrombotic therapy with warfarin (target INR 2-3) plus DAPT for 1, 6, or 12 months (706 patients). ${ }^{21}$ The primary safety endpoint, consisting of clinically significant bleeding (e.g., TIMI major plus minor), was lower in the dual pathway and reduced dose triple antithrombotic therapy groups when compared to the group receiving traditional triple antithrombotic therapy with warfarin (16.8\% in patients treated with dual pathway therapy, $18 \%$ in patients treated with reduced dose triple antithrombotic therapy, and $26.7 \%$ in patients treated with traditional triple antithrombotic therapy). Similar to WOEST there was a nonsignificant reduction in major bleeding dual pathway and reduced dose triple antithrombotic therapy groups when compared to the group receiving traditional triple antithrombotic therapy with warfarin.

The "Dual antithrombotic therapy with dabigatran after PCI in atrial fibrillation" (RE-DUAL PCI) trial randomized 2,725 patients with non-valvular AF undergoing PCI for ACS (51\%) or stable CAD to: (1) dual pathway therapy with dabigatran 110mg bid plus P2Y12 inhibitor (D110 - 981 patients), (2) dual pathway therapy with dabigatran $150 \mathrm{mg}$ bid plus P2Y12 inhibitor (D150 - 763 patients), or (3) traditional triple antithrombotic therapy with warfarin (target INR 2-3) plus a P2Y12 inhibitor (clopidogrel or ticagrelor) plus ASA (981 patients).23 In the triple antithrombotic therapy group aspirin was discontinued after 1 month (in patients with BMS) or after 3 months (in patients with DES), however the P2Y12 inhibitor was continued for 12 -months post-PCI. The primary outcome was ISTH major or clinically relevant non-major bleeding, which was significantly reduced in both dual pathway therapy groups when compared to triple antithrombotic therapy (11.5\% absolute reduction with D110 and 5.5\% absolute reduction with D150). In secondary efficacy analyses both dual pathway therapy groups significant reduced ISTH major bleeding (4.2\% absolute reduction with D110 and 2.8\% absolute reduction with D150), and TIMI major bleeding (2.4\% absolute reduction with D110 and $1.8 \%$ absolute reduction with D150). While the study was underpowered to detect differences in thromboembolic events, the rates of death, myocardial infarction, stroke, or stent thrombosis were similar across the three groups, the use of dual pathway therapy was noninferior for the composite efficacy endpoint (thromboembolic events).

There are several notable limitations to the above trials. Firstly, a large proportion of patients were undergoing elective PCI ( $72 \%$ in WOEST, $48 \%$ in PIONEER AF-PCI, and $44 \%$ in RE-DUAL), meaning the rate relative risk of coronary outcomes may be underestimated. Second, measures to decrease bleeding risk were underutilized, suggesting that the observed bleeding rate may be higher than contemporary practice. Third, it is unknown whether the results of PIONEER AF-PCI and REDUAL PCI would have been similar had the dual pathway therapy groups utilized a VKA, or if the triple therapy group utilized a standard-dose NOAC.

While each of the trials were individually underpowered to detect meaningful differences in the incidence of ischemic events a limited meta-analysis of these randomized trials demonstrated that the use of dual pathway therapy was associated with a significant reduction in major bleeding events (2.22\% vs $3.78 \%$; OR $0.58,95 \%$ CI $0.39-0.86)$, without an excess in the occurrence of MI (3.58\% vs. $3.21 \%$; OR 0.96$)$, stent thrombosis ( $1.02 \%$ vs. $0.77 \%$; OR 0.95$)$, or stroke $(1.35 \%$ vs. $1.43 \%)$.

However, the meta-analysis focused only on the comparison between dual pathway therapy and triple antithrombotic therapy. We have performed a larger network meta-analysis of 114,887 ACS patients in 10 randomized and 38 non-randomized studies to examine the relative risks/benefits of DAPT vs. dual pathway therapy versus triple antithrombotic therapy. In comparison to DAPT both dual pathway therapy and triple antithrombotic therapy were associated with a significantly higher incidence of major bleeding and with a significant reduction in stroke, but no significant effect on myocardial infarction or all-cause mortality (Figure 1). Compared to dual pathway therapy, triple antithrombotic therapy demonstrated significantly more major bleeding (OR 1.54; 95\%CI 1.18-2.00), a trend towards less myocardial infarction (OR 0.82; 95\%CI 0.66-1.03) and stent thrombosis (OR 0.66; 95\%CI 0.42-1.03), with no differential effect on stroke or all-cause mortality. 


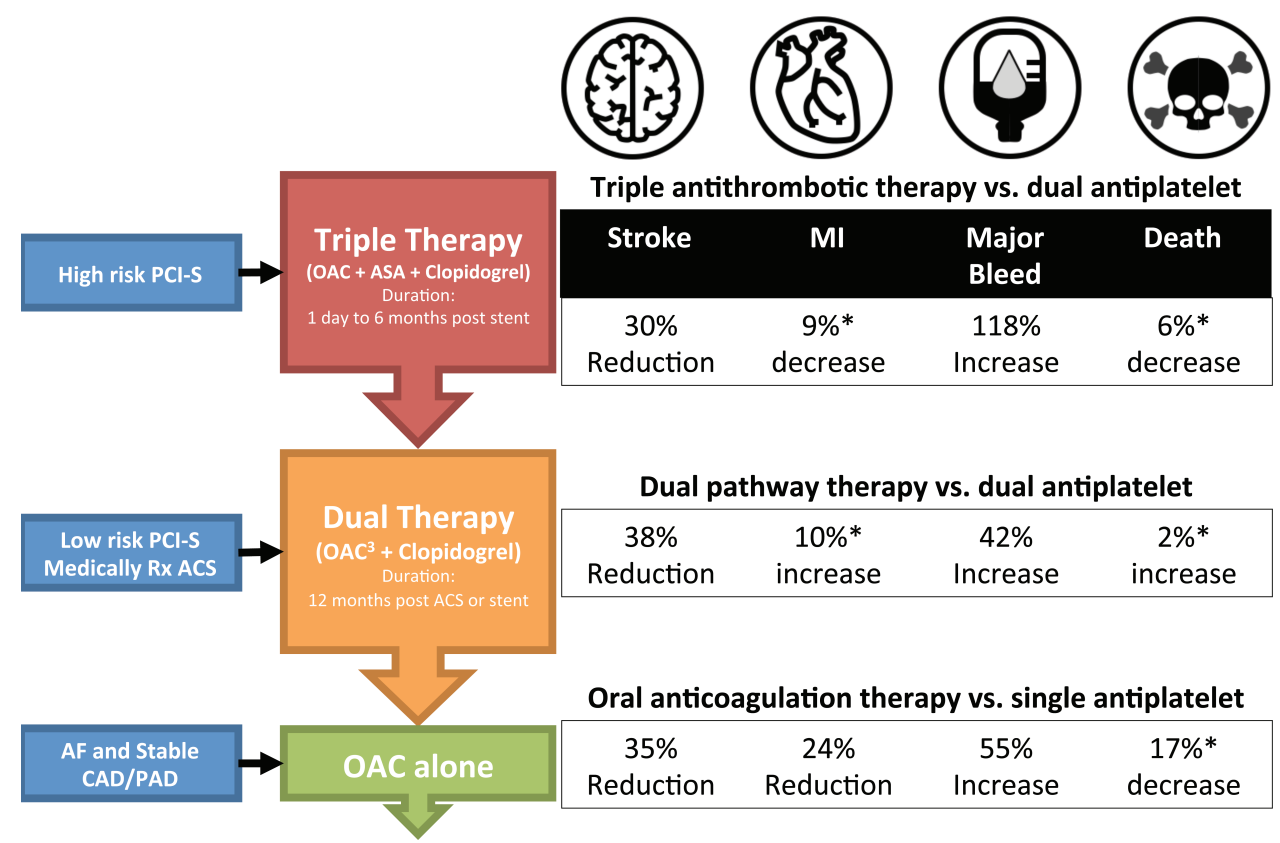

Figure 1. Relative risk and benefits of antithrombotic therapies for each treatment phase.

\section{Question 2 - Should We Use Noacs As Part of the Dual or Triple Therapy Regimen?}

It has been postulated that the use of NOACs may be beneficial given the increased risk of fatal and nonfatal bleeding events (including intracranial hemorrhage) observed with dual pathway or triple antithrombotic therapy. Collectively the phase 3 clinical trials studies comparing NOAC therapy to warfarin demonstrated a non-significant $14 \%$ reduction in major bleeding with NOAC therapy when compared to warfarin $\left(95 \% \mathrm{CI}\right.$ 0.73-1.00). ${ }^{9}$ The majority of this benefit was achieved through a significant reduction in hemorrhagic stroke, and subdural, epidural, and subarachnoid bleeding (RR 0.48; 95\%CI 0.39-0.59), which was counterbalanced by an increase in gastrointestinal bleeding (RR 1.25 ; 95\%CI 1.01-1.55).

For the subset of subjects (24-38\%; total 21,722 patients) who received combination OAC and antiplatelet therapy the use of a standard-dose NOAC was not associated with any significant difference in outcomes, however non-significant trends were observed in terms of reduction in major bleeding (OR 0.94; 95\%CI $0.80-1.10$ ), all-cause mortality (OR 0.91; 95\%CI 0.79-1.04), and stroke (OR 0.89; 95\%CI 0.62-1.30); with a non-significant increase in the rates of myocardial infarction with NOAC therapy (OR 1.24 ; $95 \%$ CI $0.98-1.57$ ) when compared to warfarin.

\section{Question 3 - What Is the Preferred P2Y12 Inhibitor As Part of the Combination Therapy Regimen?}

In patients without a need for OAC, the antiplatelet guidelines recommend the use of prasugrel or ticagrelor in preference to clopidogrel for the management of non-ST-segment elevation ACS and ST-elevation myocardial infarction. This is because of their greater efficacy at reducing recurrent MI (11\% reduction with prasugrel; $15 \%$ reduction with ticagrelor), and stent thrombosis (52\% reduction with prasugrel; $26 \%$ reduction for ticagrelor). ${ }^{10,11}$ However, these agents are not recommended as a part of a combination therapy owing to their greater propensity of bleeding. ${ }^{12}$ As such, clopidogrel is the preferred P2Y12 inhibitor when used in combination therapies with an OAC.

\section{Questions 4 - What Is the Optimal Duration of Triple Antithrombotic Therapy?}

The benefit of triple antithrombotic therapy in reducing ischemic outcomes is established in selected subgroups (i.e., those undergoing high-risk PCI), however this benefit must be balanced against the increased bleeding risk with this therapeutic regimen (Figure 2).

The "Intracoronary Stenting and Antithrombotic RegimenTesting of a 6-Week Versus a 6-Month Clopidogrel Treatment Regimen in Patients with Concomitant Aspirin and Oral Anticoagulant Therapy Following Drug-Eluting Stenting" (ISAR-TRIPLE) study randomized patients receiving OAC to 6 weeks or 6 months of triple antithrombotic therapy (307 patients in each group). ${ }^{13}$ All patients underwent PCI (33.2\% with ACS). The primary composite endpoint included death, MI, stent thrombosis, stroke, or TIMI major bleeding. Although underpowered, there was not difference in composite outcome (cardiac death, MI, stent thrombosis, or ischemic stroke - $4.0 \%$ vs. $4.3 \%$; HR 0.93, 95\%CI 0.43-2.05), TIMI major bleeding 




Figure 2. Risk factors for bleeding, stroke, and coronary ischemic events.

(5.3\% vs. $4.0 \%$; HR $1.35,95 \%$ CI $0.64-2.84)$, or death (4.0\% vs. $5.2 \%$; HR $0.75,95 \%$ CI $0.35-1.59$ ). A post-hoc landmark analysis from 6 weeks to 9 months (i.e. excluding the period where both groups were on TT) demonstrated a significant reduction in any bleeding in the 6 weeks group ( $20.5 \%$ vs. $27.9 \%$; HR 0.68 , 95\%CI 0.47-0.98). In addition, the ischemic outcomes did not differ between the $51 \%$ of patients in PIONEER AF-PCI used TT durations shorter than 12 months, compared to those who were on TT for a year. On balance, these findings suggest shortening the triple antithrombotic therapy course to $\leq 6$ months, and thereafter continuing with OAC and clopidogrel, is reasonable in patients at elevated bleeding risk.

\section{Question 5 - What Is the Optimal Antithrombotic Therapy for AF Patients With Stable CAD?}

For patients with both $\mathrm{AF}$ and $\mathrm{CAD}$ the question that dominates is whether OAC therapy alone is an adequate substitute for ASA, or whether ASA should be added to OAC therapy on the rationale that the anticoagulant effect of a VKA combined with the antiplatelet effect of ASA may enhance antithrombotic efficacy.

Historically several studies have evaluated this question. These studies randomized post myocardial infarction patients to ASA alone vs. combined warfarin and aspirin therapy, ${ }^{14-17}$ or ASA alone versus warfarin alone versus combined warfarin and aspirin therapy. ${ }^{18,19}$ Collectively these studies demonstrated that combination therapy was associated with a reduction in the combined endpoint of death/non-fatal MI/non-fatal stroke (OR $0.73 ; 95 \%$ CI $0.63-0.84)$ with a reduction in the rate of myocardial infarction (OR 0.70; 95\%CI 0.52-0.95) and stroke (RR 0.43; 95\% CI 0.27-0.70), but an increase in the risk of bleeding (OR 2.32 ; $95 \%$ CI 1.63-3.29), when compared to ASA monotherapy. ${ }^{20}$
Interestingly, OAC monotherapy was associated with similar rates of death, MI, and stroke (e.g. 16.7\% in warfarin group vs. $15.0 \%$ in Warfarin plus ASA group; $\mathrm{P}=0.18$ ) but lower rates of bleeding compared to combined OAC and aspirin therapy (e.g., overall bleeding $2.82 \%$ vs. $3.27 \%) .{ }^{18,19}$

When these studies are put together in a network meta-analysis $(132,657$ CAD patients in 13 randomized and 7 non-randomized studies) OAC monotherapy was associated with significantly less major bleeding (OR 0.76; 95\%CI 0.65-0.90), and a lower all-cause mortality (OR $0.82 ; 95 \%$ CI $0.72-0.94$ ), with a trend towards reduced MI (OR $0.84 ; 95 \%$ CI $0.70-1.00)$ vs. combination therapy. Therefore, for patients at risk of AF-associated stroke/ systemic embolism the cumulative evidence base supports the use of OAC alone as it provides protection against both stroke and coronary events. The addition of antiplatelet therapy to OAC confers an increased risk of adverse bleeding outcomes while providing only limited benefit on coronary outcomes.

\section{Summary}

General recommendations for antithrombotic therapy include the following (see Figure 1):

1. For patients at low risk of AF-associated stroke (age $<65$ years and CHADS2 Score $=0$ ) OAC therapy of the coronary generally not recommended. The disease should follow the recommendations found in the 2018 CCS/CAIC Focused Update of the Guidelines for the Use of Antiplatelet Therapy.

2. For patients at high risk of AF-associated stroke (age $\geq 65$ years or $\mathrm{CHADS}_{2}$ Score $\geq 1$ ) and stable CAD we recommend $\mathrm{OAC}$ alone. 
3. For patients at high risk of AF-associated stroke (age $\geq 65$ years or $\mathrm{CHADS}_{2}$ Score $\geq 1$ ) undergoing elective or low-risk PCI, OAC combined with clopidogrel (dual therapy) for one year should be employed.

4. For patients at high risk of AF-associated stroke (age $\geq 65$ years or $\mathrm{CHADS}_{2}$ Score $\geq 1$ ) undergoing highrisk PCI, OAC combined with ASA and clopidogrel (triple therapy) should be employed for up to 6 months post PCI, followed by dual therapy with OAC plus clopidogrel until one-year post PCI.

5. When OAC is used a NOAC is preferred.

6. Clopidogrel is the preferred antiplatelet agent in dual pathway or triple therapy.

\section{Conclusion}

The management of patients with concomitant AF and CAD is challenging and requires a comprehensive assessment of the competing risks of the therapeutic options. For most patients OAC alone can be used for stable CAD, with triple therapy used for a short period ( $<6$ months) after ACS or high-risk PCI, followed by dual therapy until 12 months post event.

\section{References}

1. Feinberg WM, Blackshear JL, Laupacis A, Kronmal R and Hart RG. Prevalence, age distribution, and gender of patients with atrial fibrillation. Analysis and implications. Arch Intern Med 1995;155:469-73.

2. Go AS, Hylek EM, Phillips KA, Chang Y, Henault LE, Selby JV and Singer DE. Prevalence of diagnosed atrial fibrillation in adults: national implications for rhythm management and stroke prevention: the AnTicoagulation and Risk Factors in Atrial Fibrillation (ATRIA) Study. JAMA 2001;285:2370-5.

3. Lip GY, Laroche C, Dan GA, et al. A prospective survey in European Society of Cardiology member countries of atrial fibrillation management: baseline results of EURObservational Research Programme Atrial Fibrillation (EORP-AF) Pilot General Registry. Europace 2014;16:308-19.

4. Kralev S, Schneider K, Lang S, Suselbeck T and Borggrefe M. Incidence and severity of coronary artery disease in patients with atrial fibrillation undergoing first-time coronary angiography. PLoS One 2011;6:e24964.

5. Connolly S, Pogue J, Hart R, et al. Clopidogrel plus aspirin versus oral anticoagulation for atrial fibrillation in the Atrial fibrillation Clopidogrel Trial with Irbesartan for prevention of Vascular Events (ACTIVE W): a randomized controlled trial. Lancet 2006;367:1903-12.
6. Eikelboom JW, Connolly SJ, Bosch J, et al. Rivaroxaban with or without Aspirin in Stable Cardiovascular Disease. N Engl J Med 2017;377:1319-30.

7. Bonaca MP, Bhatt DL, Cohen M, et al. Long-term use of ticagrelor in patients with prior myocardial infarction. N Engl J Med 2015;372:1791-800.

8. Udell JA, Bonaca MP, Collet JP, et al. Long-term dual antiplatelet therapy for secondary prevention of cardiovascular events in the subgroup of patients with previous myocardial infarction: a collaborative meta-analysis of randomized trials. Eur Heart J 2016;37:390-9.

9. Ruff CT, Giugliano RP, Braunwald E, et al. Comparison of the efficacy and safety of new oral anticoagulants with warfarin in patients with atrial fibrillation: a meta-analysis of randomized trials. Lancet 2014;383:955-62.

10. Shah R, Rashid A, Hwang I, Fan TM, Khouzam RN and Reed GL. Metaanalysis of the relative efficacy and safety of oral p2y12 inhibitors in patients with acute coronary syndrome. Am J Cardiol 2017;119:1723-28.

11. Mehta SR, Bainey KR, Cantor et al. 2018 Canadian Cardiovascular Society/ Canadian Association of Interventional Cardiology Focused Update of the Guidelines for the Use of Antiplatelet Therapy. Can J Cardiol 2018;34:214-33.

12. Sarafoff N, Martischnig A, Wealer J, et al. Triple therapy with aspirin, prasugrel, and vitamin $\mathrm{K}$ antagonists in patients with drug-eluting stent implantation and an indication for oral anticoagulation. J Am Coll Cardiol 2013;61:2060-6.

13. Fiedler KA, Maeng M, Mehilli J, et al. Duration of triple therapy in patients requiring oral anticoagulation after drug-eluting stent implantation: The ISAR-TRIPLE Trial. J Am Coll Cardiol 2015;65:1619-29.

14. Coumadin Aspirin Reinfarction Study (CARS) Investigators. Randomized double-blind trial of fixed low-dose warfarin with aspirin after myocardial infarction. Lancet 1997;350:389-96.

15. Fiore LD, Ezekowitz MD, Brophy MT, Lu D, Sacco J, Peduzzi P, Combination $\mathrm{H}$ and Mortality Prevention Study G. Department of Veterans Affairs Cooperative Studies Program Clinical Trial comparing combined warfarin and aspirin with aspirin alone in survivors of acute myocardial infarction: primary results of the CHAMP study. Circulation 2002;105:557-63.

16. Herlitz J, Holm J, Peterson M, et al. Effect of fixed low-dose warfarin added to aspirin in the long term after acute myocardial infarction; the LoWASA Study. Eur Heart J 2004;25:232-9.

17. Brouwer MA, van den Bergh PJ, Aengevaeren WR, et al. Aspirin plus coumarin versus aspirin alone in the prevention of reocclusion after fibrinolysis for acute myocardial infarction: results of the Antithrombotics in the Prevention of Reocclusion In Coronary Thrombolysis (APRICOT)-2 Trial. Circulation 2002;106:659-65.

18. Hurlen M, Abdelnoor M, Smith P, Erikssen J and Arnesen H. Warfarin, aspirin, or both after myocardial infarction. N Engl J Med 2002;347:969-74.

19. van Es RF, Jonker JJ, Verheugt FW, et al. Aspirin and coumadin after acute coronary syndromes (the ASPECT-2 study): a randomized controlled trial. Lancet 2002;360:109-13.

20. Andreotti F, Testa L, Biondi-Zoccai GG, and Crea F. Aspirin plus warfarin compared to aspirin alone after acute coronary syndromes: an updated and comprehensive meta-analysis of 25,307 patients. Eur Heart J 2006;27:519-26. 